British Journal of Industrial Medicine 1985;42:1-3

\title{
Editorial
}

\section{Which way ahead?}

Since the British Journal of Industrial Medicine was first published in 1944 great advances have been made in occupational medicine; old diseases have become much better understood-those caused by asbestos, for example-and new diseases have been recognised and their aetiology determined; one might cite as examples here vinyl chloride disease and hexacarbon neuropathy. The most important change, however, has been that the prevalence of the toxic industrial diseases and the pneumoconioses has markedly declined. This has been due in large part to better control of conditions in the workplace but also to changes in the industrial life of the country. Donald Hunter, the first Editor of the BJIM, was able to conduct ward rounds at the London Hospital during which his students were introduced to a seemingly constant flow of cases of metal and solvent poisoning, of dust diseases, and of toxic neuropathy. It was this clinical material that formed the basis for his Diseases of Occupations in which the experience gained from more than 20 years of teaching occupational medicine (for he never did work in industry) was set down for the great benefit of posterity. No occupational physician could expect to be able to draw on anything like such a crop of diseases nor would he hope to do so. An illustrations of the changes which have come about in the practice of occupational medicine is that, although first published only 30 years ago, Hunter's book is now read more as an important social document than to learn about the diseases which, commonly, are likely to afflict men and women at work. Taylor, in reviewing the sixth and final edition in 1979 , noted that many of the diseases so beautifully described belonged to history and suggested that the future of occupational medicine lay along a different path, that of evaluating and measuring small deviations from normal health. ${ }^{1}$ Lee has also noted the change in occupational medicine, commenting that its practitioners now spend most of their time dealing with the effects of health on the capacity to work rather than on the study of the effects of work on health. ${ }^{2}$

The question which is raised for discussion here is, how best is the specialty to adapt itself to its changing need? When Alice asked the Cheshire Cat which way she ought to go, the Cat replied, "That depends a good deal on where you want to get to." Occupa- tional physicians, like Alice, must decide precisely where they want to get to, for unless they know that, it will not be possible to determine their route, nor what training will be necessary to enable them to undertake the journey. The specialty is already under threat; the last reorganisation of the National Health Service left occupational physicians as the only group of doctors outside the Service and the only specialty in which the cost of training is not paid for from the public purse. In his foreword to the first issue of the BJIM, Lord Moran declared that "the industrial doctor of the future must have close links with the main chain of general medicine" and he predicted that the structure of industrial medicine would be similar to that of the other medical services in the country ${ }^{3}$; the exclusion of occupational physicians from the main body medical has put paid to that hope.

The main difficulty in defining the future role for the occupational physician seems to be in the precise determination of those skills and that body of wisdom which is uniquely his. As the old industrial diseases disappear, the expectations of workers, and of society at large, will change; it will no longer be sufficient that work does not produce clinically obvious disease, but there will be (quite properly) a demand that neither does it induce what one might call subclinical or physiological aberrations from normal, the detection of which would not be in the power of the clinician with the usual clinical means. That this is already happening can be shown by the fact that much research into occupational health is already in the hands of epidemiologists and statisticians as a glance through a few back numbers of the $B J I M$ will show; chest physicians and dermatologists deal with a considerable fraction of occupational lung and skin disease; the prevention of accidents is the remit of the safety officer; so what has the occupational physician uniquely to offer? His great strength lies in his intimate knowledge of the workplace and the job requirements of each of the workers in his care. It is thus with some alarm that we view the recommendations of the House of Lords Select Committee ${ }^{4}$ that, in future, general practitioners should be the focus of occupational health services; the committee does acknowledge, however, that participating general practitioners would 
need to learn something about the subject. This recommendation, if implemented will further undermine occupational medicine as a specialty in its own right; the appeal of the recommendation to government, however, is obvious since, unlike the provision of a nationally organised occupational health service running in parallel with the NHS, it can be provided free to employers and at almost no cost to the state.

In his thoughtful review Lee asks whether we should wait for initiatives to come from the top or begin to make regional moves. ${ }^{2}$ The answer must surely be that the time has come for all those who care about the future of occupational medicine to come to its aid, to clarify its purpose, and to demand adequate training facilities funded from the public purse, so that we can look forward to an improvement in the health and welfare of men and women at work, which in the next 40 years will be as significant

\section{Guidance for authors}

The British Journal of Industrial Medicine publishes material that is relevant to any aspect of occupational health. Papers that deal with environmental medicine will also be considered. The journal publishes four types of communication, original papers, short reports (which may include reports of clinical material), editorials, and letters to the Editor. Review articles are normally specially commissioned, and authors should not submit such a review without prior consultation with the Editor.

All material submitted to the journal must be typewritten on one side of the paper only with double spacing and wide margins. Manuscripts must be submitted in triplicate to the Editor, TUC Centenary Institute of Occupational Health, London School of Hygiene and Tropical Medicine, Keppel Street (Gower Street), London WC1E 7HT; they must conform with the recommendations given below. Manuscripts must be written in English and spelling must follow the conventions in the Oxford English Dictionary. Both SI units and their equivalents must be given throughout. Authors should note that clarity and brevity are virtues that are given great weight when considering a paper for publication. The Editor cannot enter into correspondence about papers that are rejected as being unsuitable for publication and his decision is final.

Papers are accepted on the understanding that the work described has not appeared in whole elsewhere and that they are subject to editorial revision. Where the findings have been published elsewhere as that achieved during the last 40 .

"I don't care much where," said Alice, "so long as I get somewhere."

"Oh you're sure to do that," said the Cat, "if only you walk long enough."

Occupational physicians must surely know precisely where it is they wish to go or they may find that they have not long enough to walk.

H A WALDRON

Editor,

British Journal of Industrial Medicine

\section{References}

' Taylor W. Man and his job. Br Med J 1979;i:1066-7.

${ }^{2}$ Lee WR. Medicine in the workplace. Br Med J 1984;287:1326.

${ }^{3}$ Moran W. Foreword. Br J Ind Med 1944;1:1.

${ }^{4}$ House of Lords Select Committee on Science and Technology. Occupational health and hygiene services. London: HMSO, 1983.

in part this must be clearly stated and the submitted manuscript should be accompanied by a copy of the publication that contains those findings. If part of the findings are contained in a manuscript which is under consideration elsewhere a copy of that manuscript should be included with that submitted to the journal. A letter giving consent to publication must be signed by all those whose name appears on the manuscript. Papers that describe studies carried out on human subjects must give evidence that the protocol was approved by an ethical committee and that all the subjects gave their informed consent.

There is no prescribed length for original papers but authors should not submit papers which exceed 10000 words (about 30 typewritten sheets) without first consulting the Editor.

Original papers should follow the requirements of the International Steering Committee of Medical Editors; details, with which authors should familiarise themselves, are to be found in the British Medical Journal (1979;i:532-5). Papers should be prefaced by an abstract of the argument and findings and should be more comprehensive than a summary. The abstract must not contain references. Short reports or case reports do not require an abstract.

Photographs on glossy paper should be submitted unmounted. Colour photographs will not be published unless the authors underwrite the cost of production. Charts and graphs should be carefully drawn in black ink on tracing linen, Bristol board, or stout white paper. Only one original of the figures 